\title{
Real-time plasma control in a dual-frequency, confined plasma etcher
}

\author{
V. Milosavljevićc, ${ }^{1,2, a)}$ A. R. Ellingboe, ${ }^{1}$ C. Gaman, ${ }^{1}$ and J. V. Ringwood ${ }^{3}$ \\ ${ }^{1}$ PRL, School of Physics and NCPST, Dublin City University, Dublin 9, Ireland \\ ${ }^{2}$ Faculty of Physics, University of Belgrade, P.O.B. 368, Belgrade, Serbia \\ ${ }^{3}$ Department of Electronic Engineering, NUI Maynooth, Maynooth, Co., Kildare, Ireland
}

(Received 23 November 2007; accepted 22 January 2008; published online 22 April 2008)

\begin{abstract}
The physics issues of developing model-based control of plasma etching are presented. A novel methodology for incorporating real-time model-based control of plasma processing systems is developed. The methodology is developed for control of two dependent variables (ion flux and chemical densities) by two independent controls ( $27 \mathrm{MHz}$ power and $\mathrm{O}_{2}$ flow). A phenomenological physics model of the nonlinear coupling between the independent controls and the dependent variables of the plasma is presented. By using a design of experiment, the functional dependencies of the response surface are determined. In conjunction with the physical model, the dependencies are used to deconvolve the sensor signals onto the control inputs, allowing compensation of the interaction between control paths. The compensated sensor signals and compensated set-points are then used as inputs to proportional-integral-derivative controllers to adjust radio frequency power and oxygen flow to yield the desired ion flux and chemical density. To illustrate the methodology, model-based real-time control is realized in a commercial semiconductor dielectric etch chamber. The two radio frequency symmetric diode operates with typical commercial fluorocarbon feed-gas mixtures $\left(\mathrm{Ar} / \mathrm{O}_{2} / \mathrm{C}_{4} \mathrm{~F}_{8}\right)$. Key parameters for dielectric etching are known to include ion flux to the surface and surface flux of oxygen containing species. Control is demonstrated using diagnostics of electrode-surface ion current, and chemical densities of $\mathrm{O}, \mathrm{O}_{2}$, and $\mathrm{CO}$ measured by optical emission spectrometry and/or mass spectrometry. Using our model-based real-time control, the set-point tracking accuracy to changes in chemical species density and ion flux is enhanced. () 2008 American Institute of Physics. [DOI: 10.1063/1.2903137]
\end{abstract}

\section{INTRODUCTION}

Plasmas are becoming ubiquitous for surface modification in modern manufacturing. The need to improve the consistency of these plasma processes is driving the effort for fundamental understanding of the nature of power coupling, ${ }^{1,2}$ plasma formation, plasma chemistry, ${ }^{3-5}$ and plasma surface interaction. ${ }^{6}$ For application to dielectric films, radio requency (rf) plasmas are typically used. This is particularly true in the application of plasma processing to the manufacture of semiconductors, and many different suites of hardware and associated processes have been developed to address the hundreds of processing steps used in manufacturing. The continuing drive of Moore's Law (the doubling of chip speed and capacity every 18 months) is demanding increased reproducibility and better control of the plasma process. Despite the increasing demands for reproducibility, most processes operate under open-loop control conditions, often termed "recipe control."

In the development of a recipe process, optimization is done using design of experiment (DOE) techniques ${ }^{7}$ to correlate the process outputs [etch rate, critical dimension (CD), photoresist pull back, uniformity, etc.] against process input variables (rf power, pressure, and gas flows). In general, black-box evaluation of the DOE, coupled with process experience, is used to determine the optimized process condition.

\footnotetext{
${ }^{a)}$ Author to whom correspondence should be addressed. Electronic mail: vladimir@physics.dcu.ie and vladimir@ff.bg.ac.yu.
}

Key variables for the etching of dielectric films include ion energy, ion flux, and surface fluxes of chemical species. High-aspect-ratio-contact etch (where holes are etched through layers of dielectric to allow electrical contact between layers of conductors) is one of the most demanding processes. The acceptable process window can be very small; it can be such that "etch stop" (the etch does not reach the bottom conductor) occurs for a given flow of $\mathrm{O}_{2}$ (e.g., 15 $\mathrm{sccm}$ ) and increasing the flow by a very small amount (e.g., to $16 \mathrm{sccm}$ ) results in "blow out" (where neighboring holes are not distinct at the top conductor surface or significant loss of $\mathrm{CD}$ ). Thus, the reliance on open-loop recipes and process knowledge, in the face of material and environmental uncertainty, is suboptimal in achieving consistent product.

Drifts in a plasma process occur due to a large variety of sources. Drifts in oxygen density can be caused by changes in surface and gas-phase chemistry caused by changes in surface temperature, pressure and particle density chamber history, wear, erosion and deposition on the plasma facing surfaces, water absorption by the wafer surface, or inconsistencies in helium-cooling flow. In addition, the etching of dielectric films releases large quantities of oxygen into the discharge; increased etch rate increases oxygen density which reduces the deposition rate of carbon onto the oxide surface which, in-turn, further increases the etch rate.

The high sensitivity of these processes to uncontrollable (and usually unmeasurable) disturbances in the process makes dielectric etching an ideal candidate for the application of real-time plasma control. 


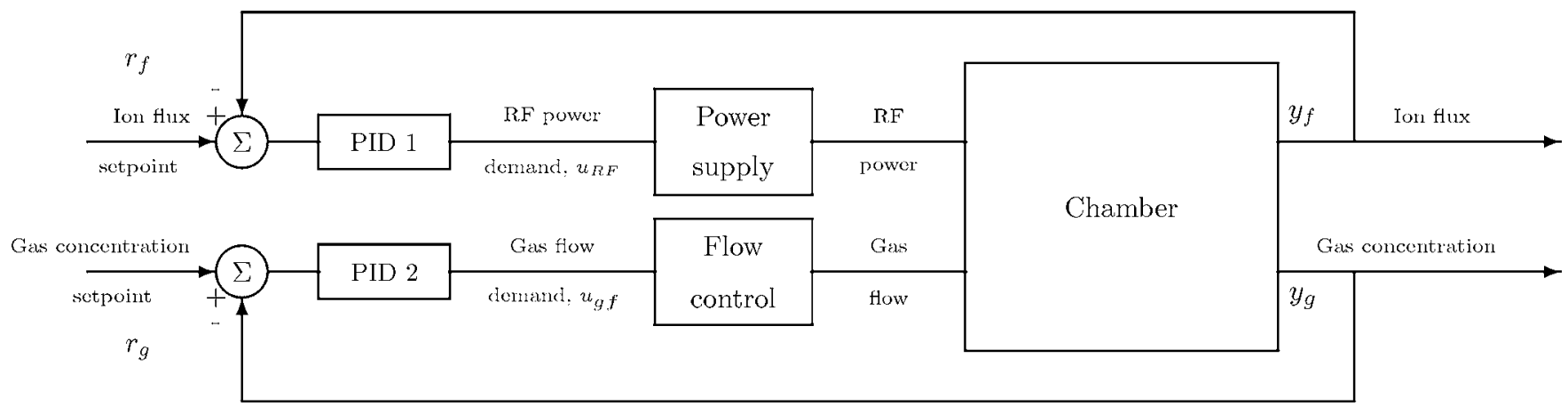

FIG. 1. System block diagram.

\section{REAL-TIME PROCESS CONTROL WITH PLASMA VARIABLES}

Real-time plasma control for surface modification of materials is a challenging problem. The system is multivariate, multitime scale, time varying, and nonlinear. Typical recipe control setup provides for setting of approximately 100 inputs. The chamber parts wear during processing, with a scheduled maintenance time scale of thousands of hours. Substrate lots (typically "boats" of 13 wafers) are processed sequentially with variable time between lots. This time scale is typical of the chamber thermal time constants of several minutes. The plasma chemistry is, largely, driven by the plasma electrons in Maxwellian-like distributions with a characteristic energy of a few electron-volts $(\mathrm{eV})$; with thresholds for both ionization and chemical reactions of a few to tens of $\mathrm{eV}$, the reaction rates are very sensitive to the exact electron temperature, and to any deviation from Maxwellian distributions.

A number of real-time control schemes for plasma-based processes have been considered. A number are documented in the excellent review by Edgar $e t a l^{8}$ and papers by Butler et $a .^{9}$ and Rashap et al. ${ }^{10}$ describe attempts to implement feedback control on wafer variables, such as etch rate, directly. Our approach is to consistently control plasma variables, which might enable etch recipes (for example) to be specified in terms of plasma variables, rather than manipulated variables, such as rf power and gas flow rates.

Our focus, in this paper, is on real-time intrawafer control, rather than addressing inter wafer and lot-to-lot variations, which are better addressed by run-to-run or statistical process control. Such longer-term control strategies contrast with continuous control and would require installation in a manufacturing environment for evaluation and are beyond our scope.

To demonstrate the methodology for development of plasma control suitable for a manufacturing environment, we investigate independent control of two outputs via variation of set points of two independent inputs. We use proportionalintegral-derivative (PID) loops to vary the set point of the independent variables. The setup is as shown in Fig. 1.

As a first example of two parameter control we address control of oxygen density and ion flux via independent manipulation of $27 \mathrm{MHz}$ rf power (P27) and $\mathrm{O}_{2}$ gas flow. In the case of a plasma chemistry of argon/oxygen gas mixtures, the two controlled variables (of ion flux and atomic $\mathrm{O}$ ) can be (almost exactly) separately mapped onto the manipulated variables; the mapping is found to be nearly orthogonal, and the $27 \mathrm{MHz}$ rf power controls the ion flux, with the $\mathrm{O}_{2}$ flow controlling the oxygen density in the discharge.

With the introduction of fluorocarbon gas into the discharge, there is a significant crosscoupling between the manipulated variables and controlled variables. The two independent controls no longer map orthogonally onto the outputs, i.e., increasing the P27 leads to an increase in ion flux and a change in the amount of oxygen in the chamber due to carbon-oxygen chemistry. Therefore, a new approach is needed. There are two ways to decouple this cross dependence:

(1) A model based-control using a physics approach, or

(2) an approach based on statistical (black box) analysis.

The development of an a priori physics-based model from a full understanding of the processes in the system is, at this point, beyond the reach of current state of knowledge. Some of our limits include the fundamental understanding of the plasma chemical kinetics, a limited understanding of the surface chemistry, particularly where the surface is a dynamic, evolving surface. In addition, the typical manufacturing optimizations of small gas addants require too much new physics to be understood for them to be applied in industry (i.e., the addant of $1 \%$ of $\mathrm{CO}$ to the feed gas would require a substantial redevelopment of the plasma chemistry to understand how and why that changes the process).

Our approach is between the two extreme ways, i.e., to develop model-based control based on simple, phenomenological based understanding of the plasma system. From the physical description, a model structure for the coupling between the inputs and outputs is developed. Using statistical analysis, the parametrization of the coupling terms can be completed.

In particular, a model relating the system outputs to the set points is specified as

$$
\hat{y}_{g}=a_{1} r_{g}+a_{2} r_{f}+a_{3} r_{g}^{2}+a_{4} r_{f}^{2}+a_{5} r_{f} r_{g}=f\left(r_{g}, r_{f}\right),
$$

where $y_{g}$ represents the value measured by the diagnostic, and $r_{g}$ and $r_{f}$ represent the set points for gas concentration and ion flux, respectively. Coefficient $a_{1}-a_{5}$ are fitting parameters which describe the interaction within the plasma system. The model in Eq. (1) can be physically justified as follows: We know, in $\mathrm{Ar} / \mathrm{O}_{2}$ discharges, that the control of 


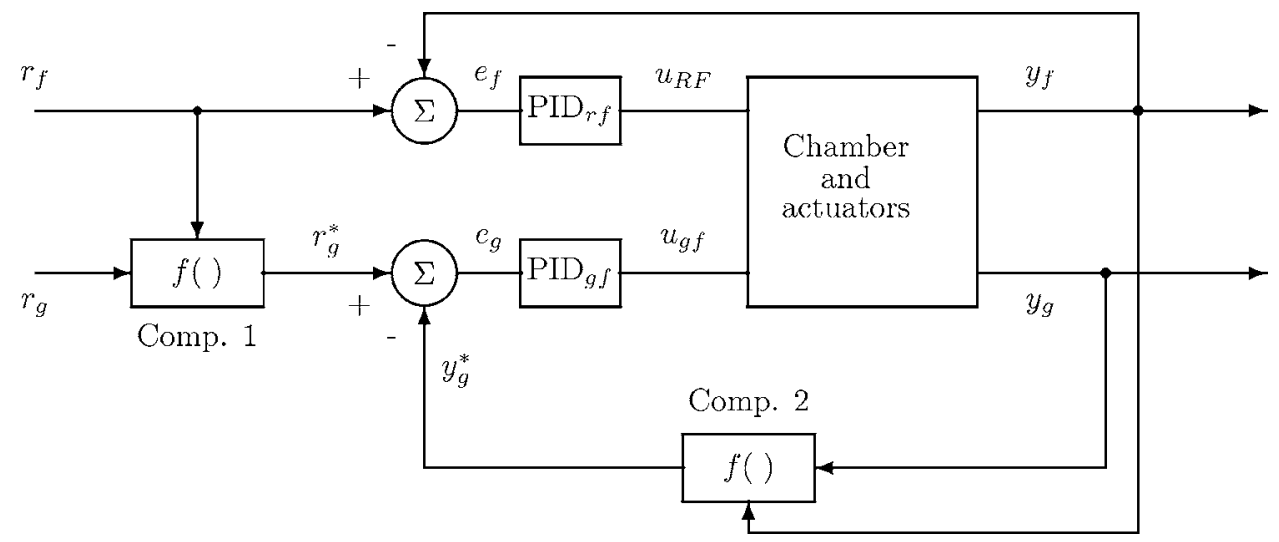

FIG. 2. Control system implementation.

power and $\mathrm{O}_{2}$ flow are independent and the response vectors are orthogonal, therefore in Eq. (1) coefficients $a_{2}-a_{5}$ have a value of zero. However, with fluorocarbon gases, we expect additional effects, e.g., an increase in the power will increase carbon dissociation, increasing $\mathrm{CO} / \mathrm{CO}_{2}$ production which leads to a reduction of oxygen density. And, since the carbon dissociation is expected to scale with ion density and ion flux, therefore Eq. (1) coefficient $a_{2}$ would be expected to have a negative sign. A similar equation for $y_{f}$ to account for interaction between gas flow and ion flux. For example, an increase of $\mathrm{C}_{4} \mathrm{~F}_{8}$ flow will increase the energy cost per electron-ion pair, reducing plasma density.

By modeling plasma parameters over a desired operational space, we create a simple physics based model which includes five terms. This is a polynomial equation with the following terms: a measurement of chemical species density, a measurement of ion flux, the square of the first term, the square of the second term, and multiplication between the first and second term.

The model is bilinear in form and can also be recognized as a low-order Volterra model. ${ }^{11}$ One of its important attributes is that it is linear in the parameters $a_{1}, a_{2}, \ldots, a_{5}$, which allows the statistical determination of these parameters using a wide variety of techniques.

Note that we specify this "interaction" dependence only for the chemical density (from rf power) since, in the main, gas flow does not significantly affect ion flux. The various chemical densities affected by these cross-coupling terms include oxygen density in $\mathrm{Ar} / \mathrm{O}_{2}$ plasma and $\mathrm{O}$ and $\mathrm{CO}$ densities in $\mathrm{Ar} / \mathrm{O}_{2} / \mathrm{C}_{4} \mathrm{~F}_{8}$ plasma.

The procedure to implement our decoupling controller is as follows:

(1) A DOE is performed in order to elicit the steady-state response of the system to a series of set points in ion flux and chemical concentration.

(2) From the DOE, $f^{*}\left(y_{f}, y_{c}\right)$, which specifies the relationship between actual outputs achieved for a given chemical composition setpoint, is determined.

(3) The relationship $r_{f}^{*}=f^{*}\left(r_{f}, r_{c}\right)$ is now directly used to modify the chemical concentration setpoint, so that $r_{f}$ and $r_{c}$ are actually achieved.

(4) The steps (1) and (2) can be repeated for a range of operating conditions, so that an appropriate function $f^{*}$ can be switched in for the given operating point (though we use the same $f^{*}$ for all 16 DOE conditions in our analysis).

While it is somewhat unusual to measure the multivariable interaction in closed loop, it does have the advantages of:

- Giving a natural steady-state solution to the decoupling problem, since set points are specified as steadystate values, and

- facilitating a simple implicit control calculation, where the adjustment to the setpoint is directly calculated from the DOE.

The alternative of (explicitly) calculating the crosscoupling on the open loop system would result in the need for an inversion of the cross-coupling term, which involves extra complexity and can be numerically sensitive. The use of a steady-state decoupling follows the general philosophy employed in the "classical" multivariable control design, using tools such as the inverse Nyquist array. ${ }^{12}$

For the purpose of closed-loop control of ion flux and chemical concentration, P27 and gas flow are considered as the primary manipulated variables. All other "input" variables (including $2 \mathrm{MHz}$ power, $\mathrm{C}_{4} \mathrm{~F}_{8}$ flow, Ar flow, chamber pressure, and He pressure), are considered as disturbances, but form the "schedule" by which the appropriate parameter values of Eq. (1) can be recalled.

As shown in Fig. 2, the "decoupling" compensator is applied in two positions in the feedback system. In steady state, the decoupling precompensator "Comp. 1," operating on $r_{f}$ and $r_{g}$, performs a static (steady-state) decoupling of the system, while "Comp. 2," operating on $y_{f}$ and $y_{g}$, performs a decoupling based on the current process state. One of the pleasing intuitive aspects of the control configuration is the fact that the dynamic control action (due to proportional and derivative terms) in the gas flow path disappears when the set points are achieved, i.e., $y_{g}^{*}=r_{g}^{*}$, when $y_{f}=r_{f}$ and $y_{g}=r_{g}$, resulting in $e_{g} \rightarrow 0$.

\section{EXPERIMENTAL METHOD}

\section{A. Plasma chamber}

Experiments were conducted in a modified Exelan ${ }^{\circledR}$ chamber (manufactured by Lam Research Corporation incorporating Lam's proprietary DFC@ plasma confinement tech- 


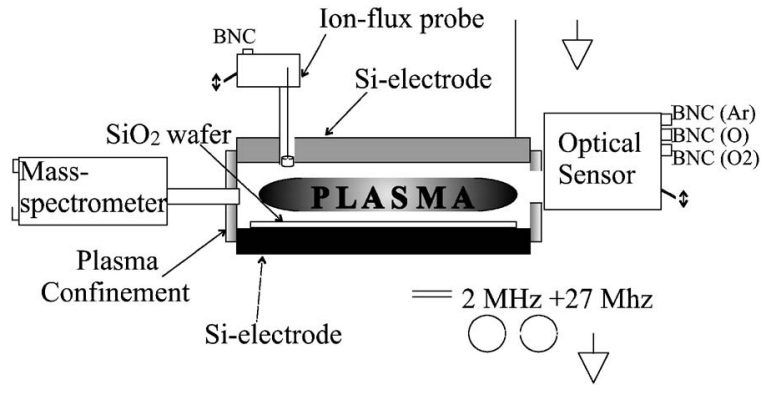

FIG. 3. Schematic diagram of the chamber with diagnostics tools.

nology). The discharge chamber is equipped with two parallel plate electrodes. The top electrode is made from single crystal silicon, and the bottom electrode is an electrostatic chuck (ESC) covered with an oxide coated silicon wafer. Electrostatic forces are used to "clamp" the wafer onto the chuck. Helium gas is admitted into the gap between the ESC and the wafer, increasing heat transfer from the wafer to be removed, thus minimizing thermal damage to the wafer. The Exelan ${ }^{\circledR}$ has a symmetric electrode configuration and uses two $(2 \mathrm{MHz}+27 \mathrm{MHz})$ capacitively coupled rf power sources which act on the driving electrode and are separated from the grounded electrode by a narrow gap of approximately $1 / 2$ in. The plasma potential $\left(V_{p}\right)$ oscillates predominantly at the $2 \mathrm{MHz}$ rf voltage with an associated highfrequency oscillation at $27 \mathrm{MHz}^{2,13}$ The peak to peak voltages can exceed $1 \mathrm{kV}$ with the mean time-averaged $V_{p}$ reaching several hundreds of volts above ground. The experimental setup is shown schematically in Fig. 3.

For the work presented here, the operating conditions involve a working gas containing a mixture of argon (Ar), oxygen $\left(\mathrm{O}_{2}\right)$, and cyclic-octafluorocyclobutane-RC318 (c $\left.-\mathrm{C}_{4} \mathrm{~F}_{8}\right)$ in a flowing regime. The gas mixture is introduced into the chamber through a showerhead in the top (grounded) electrode. Gas flows through the plasma chamber radially and is pumped out between "confinement rings" into a pump channel which is connected to a helical-groove pump via a high-conductance pumping plenum. The operating pressure of the plasma volume varies from 14.5 to $18.5 \mathrm{~Pa}$ (110 to 140 mTorr), while the corresponding flow rate of Ar is from 200 to $350 \mathrm{sccm}$. $\mathrm{O}_{2}$ and $\mathrm{C}_{4} \mathrm{~F}_{8}$ flows both range from 1 to $20 \mathrm{sccm}$, the gas flows being controlled by massflow controllers. Constant plasma area pressure for the varying reactive gas flows is achieved by varying the conductance of the confinement rings. Helium, with a changeable pressure from 0.67 to $1.33 \mathrm{kPa}$ (5 to 10 Torr), is maintained on the backside of the wafer, resulting in helium leakage into the chamber of 2-4 sccm.

The power of the $2 \mathrm{MHz}$ power generator is adjustable from 200 to $600 \mathrm{~W}$, while the power of second power generator $(27 \mathrm{MHz})$, used for feedback control of ion flux (in the configuration of Fig. 2), is operated within the limits of 50-1200 W.

\section{B. Optical diagnostic}

Optical signals are collected using photodiodes in conjunction with very narrow bandwidth optical filters. The

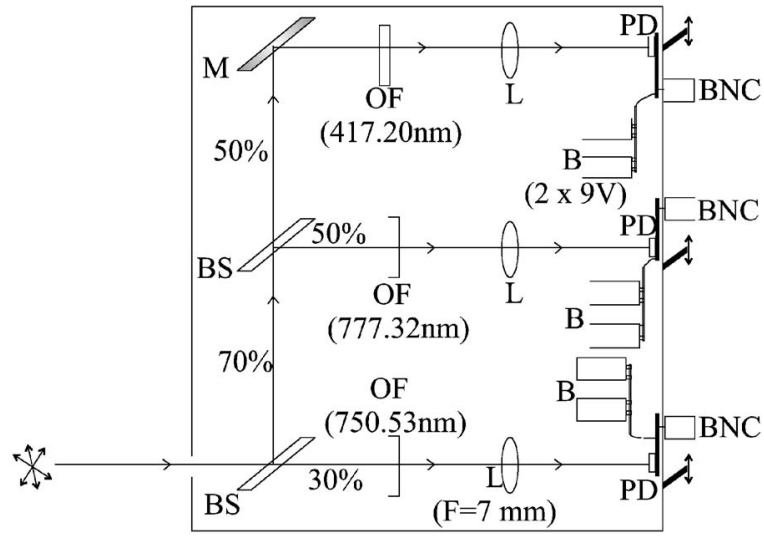

FIG. 4. A block diagram with components used for OES diagnostics: BSbeam splitter, $\mathrm{M}$-mirror, OF-optical filter (central wavelength), L-lens (focal distance), B-batteries, and PD-photodiode.

block diagram of this device is shown in Fig. 4. Using beam splitters of $70 \% / 30 \%$ and $50 \% / 50 \%$ the polychromatic light is divided into three approximately equal intensity beams. The first beam falls onto the optical filter with a central wavelength of $750.53 \mathrm{~nm}$ (the bandwidth is $0.67 \mathrm{~nm}$ and peak transmission gain is $77 \%$ ). The resulting monochromatic light then falls on the photodiode (Hamamatsu S2833$01)$. The second beam falls onto an optical filter with a central wavelength of $777.32 \mathrm{~nm}$ (the bandwidth is $0.71 \mathrm{~nm}$ and peak transmission gain is $74 \%$ ), with the resulting monochromatic light falling on a photodiode (Hamamatsu S283301). Finally, the third beam falls on a silver mirror with high reflectivity $(>98 \%)$ and passes through an optical filter with a central wavelength of $417.20 \mathrm{~nm}$ (the bandwidth is 0.57 $\mathrm{nm}$ and peak transmission gain is $64 \%$ ), with the resulting monochromatic light falling on a photodiode (Hamamatsu S5493-01). In this way, a form of spectrometer is realized, with the analog signals from each photodiode being fed to the computer. To enable direct comparison between the direct current (dc) signals from different photodiodes and optical filters, a relative calibration of the optical system is performed. The calibration ${ }^{14}$ has been performed using a Gigahertz-Optik BN-0102-1 reference standard source. The BN-0102-1 is specifically designed as a spectral radiance standard. Its tungsten halogen lamp is current controlled by the LCRT-2000 power supply. The spectral radiance calibration is supplied by the Gigahertz-Optik's calibration laboratory for a wavelength range of 380-1100 nm, in $10 \mathrm{~nm}$ increment steps. After the calibration of the optically derived signals, it is possible to directly compare signals to each other or to accurately calculate the ratio of signals. The biggest advantage of this optical box over a spectrometer is a significant reduction in complexity. The optical box does not require acquisition software or drivers which inevitably slows down signal transfer and this is undesirable for real time measurement. The output signal from the optical box is already analog so do not require the conversion. Also, the resolution of optical filters in the optical box is better then many low resolution spectrometers.

The chosen central wavelength for the optical filters $(750.5,777.32$, and $417.20 \mathrm{~nm})$ correspond to actinometry spectral lines of atomic argon $(750 \mathrm{~nm})$, atomic oxygen 
(777 nm), as well as the molecular oxygen bandhead $(417 \mathrm{~nm}) .{ }^{15}$ Signals are interpreted to give chemical species densities using actinometry with $\mathrm{Ar}$ as the actinometer. Since, in our plasma, argon is the dominant gas and, at the same time, we use argon as the actinometer, we employ mass spectroscopy as an independent technique to determinate/ control the density of oxygen.

The actinometric technique using the classical atomic oxygen (O I) spectral line representing the $3 s-3 p$ transition and from ${ }^{5} \mathrm{~S}^{o}-{ }^{5} \mathrm{P}$ multiplet, i.e., the $777 \mathrm{~nm}$ spectral line and the Ar I spectral line from $4 s-4 p$ transitions and from ${ }^{2}[1 / 2,3 / 2]^{o}-{ }^{2}[1 / 2]$, i.e., the $750 \mathrm{~nm}$ spectral line. It is important to point out that the $\mathrm{O}$ I spectral line at $777 \mathrm{~nm}$ is not just one line, but rather three lines: a $777.194 \mathrm{~nm}$ multiplet representing ${ }^{5} \mathrm{~S}_{2}^{o}-{ }^{5} \mathrm{P}_{3}$, a $777.417 \mathrm{~nm}$ multiplet representing ${ }^{5} \mathrm{~S}_{2}^{o}-{ }^{5} \mathrm{P}_{2}$ and a $777.539 \mathrm{~nm}$ multiplet representing ${ }^{5} \mathrm{~S}_{2}^{o}-{ }^{5} \mathrm{P}_{1}$. A similar situation arises in the case of the Ar I spectral line at $750 \mathrm{~nm}$, in that there are two Ar I spectral lines: A 750.387 $\mathrm{nm}$ multiplet representing ${ }^{2}[1 / 2]_{1}^{o}-{ }^{2}[1 / 2]_{0}$ and a 751.465 $\mathrm{nm}$ multiplet representing ${ }^{2}[3 / 2]_{1}^{o}-{ }^{2}[1 / 2]_{0}$. We record the spectrum of the O I $777 \mathrm{~nm}$ spectral line and the Ar I $750 \mathrm{~nm}$ spectral line using a high resolution Carl Zeiss PGS-2 spectrometer with a resolution of $60 \mathrm{pm}$, for various experimental conditions. In this way, we get three well-separated atomic oxygen spectral lines and two well-separated argon spectral lines. In attempting to understand the behavior of each $\mathrm{O} I$ spectral line in the triplet and both argon lines in the doublet, we can conclude that (for our experimental condition) the three O I spectral lines have very similar behavior (in $96 \%$ of experimental conditions), i.e., a consistent increase or decrease in each of the lines. A similar conclusion is made for each of the two argon spectral lines from the doublet. In practice, the optical box (or low resolution spectrometer) may be used for actinometry measurement, but the measured intensity of the atomic oxygen "triplet spectral line" must be multiplied by 0.31 in order to obtain the actual intensity of a single actinometry spectral line of atomic oxygen. In the case of argon, the total intensity of the doublet must be divided by factor of 0.41 so that value can be use for actinometry purposes. ${ }^{15}$

Since argon is dominant in our plasma, the validity of our actinometry is also tested. For lower concentrations of argon, the data from mass spectroscopy and actinometry show good agreement. With higher concentrations of argon, we use only mass-spectroscopy data for the determination of a species concentration. In particular, the fact that the ratio of spectral line intensities $\left(\mathrm{I}_{777} / \mathrm{I}_{750}\right.$ and $\left.\mathrm{I}_{417} / \mathrm{I}_{750}\right)$ is well correlated with the concentration of $\mathrm{O}_{2}$ is interpreted by considering that the most important mechanism of production of the 777 oxygen spectral lines is dissociative excitation, when dissociation rates are lower than $10 \%$. Reference 16 proves the validity of actinometry in $\mathrm{rf} \mathrm{O}_{2}-\mathrm{CF}_{4}$ plasmas with an applied electric field. In Ref. 17, it is shown that actinometry can be used to monitor the atomic concentration in $\mathrm{O}_{2}$ and $\mathrm{O}_{2}-\mathrm{N}_{2}$ low-pressure (67-266 Pa) microwave plasmas.

The absolute calibration/accuracy of the chemical species density by actinometry is irrelevant for the purposes of real-time control. This fact greatly enhances the usability of our technology for plasma control.

\section{Mass spectrometer}

The mass spectrometer used in this experiment is the PRISMA $80 ®$ quadrupole mass spectrometer from Pfeiffer Vacuum. The detector mass range is from 1 to $199 \mathrm{amu}$, with a detection limit of $1 \times 10^{-12}$ mbar and a resolution of 0.5 amu. The mass spectrometer is differentially pumped, using a built-in turbomolecular pump, to a working pressure of $10^{-7}$ mbar. The mass spectrometer is connected to a computer via an RS-232 serial cable and is controlled and configured using the QADSTAR $®$ software. Analog signals for up to four selectable gasses are provided with a signal bandwidth of $2 \mathrm{kHz}$.

\section{Ion flux probe}

A key parameter in the control of plasma etching (surface treatment) is the flux of energetic ions. To measure the ion flux to the surface, a small section ( $5 \mathrm{~mm}$ diameter) of the grounded upper electrode was removed. Into this void, a carbon cylinder and a thin-walled alumina cylindrical annulus was placed, such that the surface height of both the carbon and alumina are coplanar with the silicon electrode. The alumina serves to act as an electrical insulator, while the carbon slug acts as a collection surface. The collector is biased to $-18 \mathrm{~V}$ with respect to the grounded silicon electrode. Ions exiting the plasma are accelerated by the timeaverage sheath (plus the $18 \mathrm{~V}$ dc bias) onto the ion-flux probe. Electrons are repelled from the electrode by the same instantaneous potential. The collected current is, thus, only ion current, and is a measure of the ion flux delivered to the substrate (ignoring secondary electrons).

The electron density in this chamber has been determined by a hairpin probe and is nominally about $10^{10} \mathrm{~cm}^{-3} \cdot{ }^{13}$ However, implementing such a probe in a manufacturing environment would be very challenging, whereas the ion-flux probe used herein is compatible with the semiconductor manufacturing environment.

\section{E. Control algorithm implementation}

The control algorithm is implemented using a computer equipped with an analog input/output card (National Instruments PCI-6036E) running LABVIEW software. Analog signals from either the mass spectrometer or the custom optical box return the chemical species density as analog signals. Ion-flux current is measured across a resistor, and the resultant analog voltage is measured by the computer. The mathematical manipulation of the measured values results in desired set points for the independent variables, i.e., P27 and oxygen flow. The analog outputs from the input/output card are fed directly to the set-point inputs of the P27 supply and the oxygen mass-flow controller.

There is a time delay of $4-7 \mathrm{~s}$ between a change of gas flow and arrival of the gas at the chamber. The delay is due to a gas line length of $8 \mathrm{~m}$ from gas box to the chamber. This length is typical for an industrial installation. 
TABLE I. Process space DOE which approximates the operational region used in development of generic recipes (Ref. 7).

\begin{tabular}{cccccc}
\hline \hline $\begin{array}{c}\text { Operating } \\
\text { point }\end{array}$ & $\begin{array}{c}2 \mathrm{MHz} \text { power } \\
(\mathrm{W})\end{array}$ & $\begin{array}{c}\mathrm{C}_{4} \mathrm{~F}_{8} \text { flow } \\
(\mathrm{sccm})\end{array}$ & $\begin{array}{c}\text { Ar flow } \\
(\mathrm{sccm})\end{array}$ & $\begin{array}{c}\text { Pressure } \\
(\mathrm{mT} \text { Torr })\end{array}$ & $\begin{array}{c}\text { He pressure } \\
\text { (Torr) }\end{array}$ \\
\hline 1 & 200 & 1 & 250 & 100 & 10 \\
2 & 200 & 4 & 350 & 135 & 7 \\
3 & 200 & 12 & 240 & 100 & 9 \\
4 & 200 & 20 & 290 & 126 & 5 \\
5 & 300 & 1 & 290 & 114 & 9 \\
6 & 300 & 4 & 240 & 100 & 5 \\
7 & 300 & 12 & 350 & 145 & 10 \\
8 & 300 & 20 & 200 & 197 & 7 \\
9 & 500 & 1 & 350 & 134 & 5 \\
10 & 500 & 4 & 250 & 106 & 9 \\
11 & 500 & 12 & 290 & 122 & 7 \\
12 & 500 & 20 & 240 & 112 & 10 \\
13 & 600 & 1 & 240 & 98 & 7 \\
14 & 600 & 4 & 290 & 120 & 10 \\
15 & 600 & 12 & 250 & 114 & 5 \\
16 & 600 & 20 & 350 & 110 & 9 \\
\hline \hline
\end{tabular}

\section{RESULTS AND DISCUSSION}

\section{A. Control across the process space}

To cover the variety of possible recipe steps, the plasma process is run over a wide range of operating conditions. A 16-point DOE shown in Table I is used to scan the system at a range of operating points, ${ }^{7}$ as per the procedure outlined in step (4) of Sec. II. The variety of pressure values show the actual pressure values achieved under closed-loop pressure control. However, it was found that the range of variation in the model parameters across the process space did not justify the complexity of the storage of a full set of model parameters and a single model was therefore employed.

Within the steady-state operating condition of each of these process DOE points, a further DOE (shown in Table II) was performed on gas and ion flux set points, in order to estimate the "interaction function" of Eq. (1), using the configuration of Fig. 1. The duration of each of the steps of this DOE was $20 \mathrm{~s}$ and represents the typical processing time, allowing the system to achieve steady state and allowing a "static" interaction function to be identified from the data. This "inner" DOE is repeated for each species $\left(\mathrm{O}, \mathrm{O}_{2}\right.$, or $\mathrm{CO})$, giving three different "interaction" functions for each system operating point and therefore 48 functions in total. For a given gas control input $\left(\mathrm{O}, \mathrm{O}_{2}\right.$, or $\left.\mathrm{CO}\right)$, we can interpolate across the range of the 16 function parameter values to give a smooth and accurate interaction function representation across the full operational space.

Experimental results for one example experimental run (of 16 in total) and for the all 14 set points is shown in Fig.
5. The top graph in Fig. 5 shows the set point $\left(r_{f}\right)$ and the measured signal for ion flux $\left(y_{f}\right)$, while the bottom graph presents the set point $\left(r_{g}\right)$ and measured signal from mass spectroscopy $\left(y_{g}\right)$. It can been seen from Fig. 5 that the agreement between the set point and measured values is, in principle, good. In particular, the ion flux response is practically instantaneous, due to the fast power circuit dynamics. Also, the signal from the ion-flux probe is very stable except for two of the conditions.

The response of the mass-flow controller for oxygen has a delay of approximately $6 \mathrm{~s}$ (see Sec. III E), due to the $8 \mathrm{~m}$ length of gas pipe between the measured point (at the chamber) and control point (at the gas box). Note that all gases share the same pipe from gas box to the chamber and this arrangement and gas transport delay is typical of many industrial plasma tools and is therefore retained in our experimental setup.

In this $6 \mathrm{~s}$ gas transport delay period, the ion probe has already reached its set-point value. After $6 \mathrm{~s}$, the density of oxygen approaches its set point, but the achievement of steady state is affected by the interaction caused by changes in ion flux at, for example, 75 and $115 \mathrm{~s}$ (point A). This highlights the crosscoupling which occurs between ion flux and gas concentration control loops and can only be effectively corrected via the model of Eq. (1). The effective decoupling which is achieved by the control configuration of Fig. 2 is employed to remove the interaction between the process outputs.

TABLE II. DOE for estimation of interaction function. Units for $r_{g}$ and $r_{f}$ are in volts, proportional to gas density and ion flux, respectively.

\begin{tabular}{|c|c|c|c|c|c|c|c|c|c|c|c|c|c|c|}
\hline $\begin{array}{l}\text { Operational } \\
\text { points }\end{array}$ & 1 & 2 & 3 & 4 & 5 & 6 & 7 & 8 & 9 & 10 & 11 & 12 & 13 & 14 \\
\hline$r_{g}$ & 0.02 & 0.02 & 0.1 & 0.1 & 0.35 & 0.35 & 0.5 & 0.5 & 0.62 & 0.62 & 0.9 & 0.9 & 1.0 & 1.0 \\
\hline$r_{f}$ & 0.01 & 0.3 & 0.12 & 0.19 & 0.1 & 0.3 & 0.01 & 0.24 & 0.06 & 0.1 & 0.06 & 0.12 & 0.19 & 0.24 \\
\hline
\end{tabular}




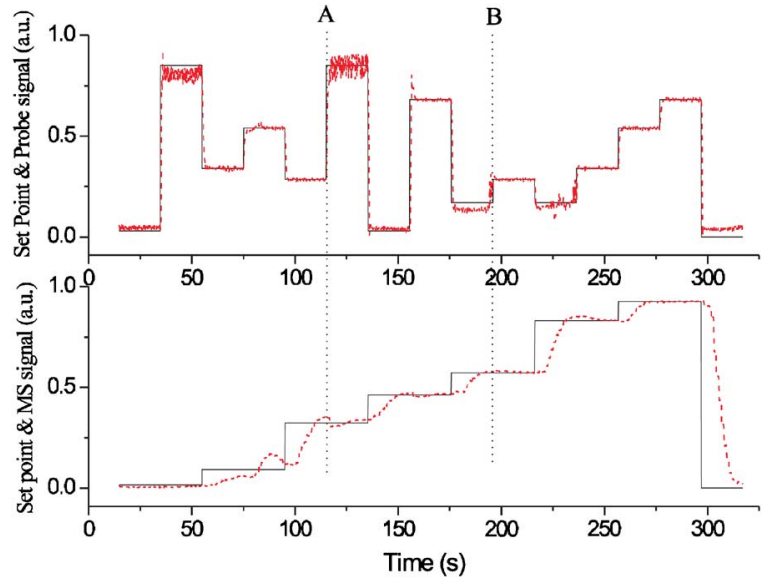

FIG. 5. (Color online) Real-time control of atomic oxygen (lower) and ion flux (upper) (operating point No. 5 in Table I). The full line represents the set point with the broken line representing the measured value. Values, in volts, are arbitrary units proportional to ion flux and gas density.

In Fig. 5, dynamic coupling between the variables $y_{f}$ and $y_{g}$ can be seen, for example, at position "A." While $r_{g}$ remains constant, the $+120 \%$ change in $r_{f}$ results not only in increased electron density, but also causes an increase in chemical activity with the extra consumption of $\mathrm{O}$ from the gas phase. The full description of the very complex chemical balance equations is extremely challenging to model in detail. A simplified chemical balance reaction like

$$
e+\mathrm{C}_{4} \mathrm{~F}_{8}+\mathrm{O}_{2} \rightarrow \ldots \rightarrow \mathrm{CO}+\text { other products }
$$

can be envisioned (i.e., a multistep reaction mechanism). Increases in left-hand side constituents leads to increases of right side densities. For example, in Fig. 5 at A, the reduction in measured $\mathrm{O}$ density occurs within the time frame of the change in $r_{f}$ (ion flux), so it is not caused by changes in gas flow. A different interaction can be seen approaching point "B," where the slow time scale change in $y_{g}$ prevents $y_{f}$ from reaching the set point, $r_{f}$. For reference, the root-meansquare (rms) errors in ion flux and species concentration, using this (naive) control configuration, are 0.0134 and 0.0621 , respectively.

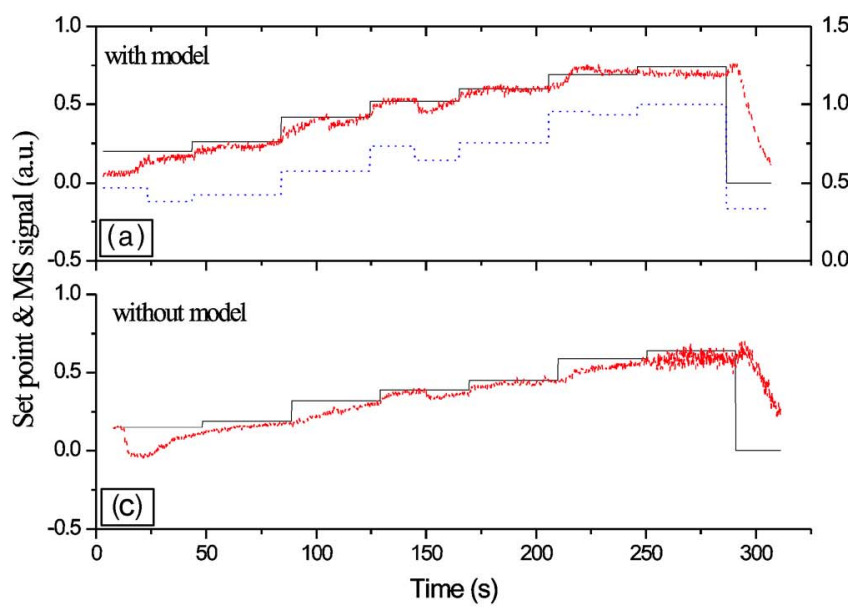

\section{B. Real-time control results}

From the interaction model, we know in $\mathrm{Ar} / \mathrm{O}_{2}$ discharges that the control of power and $\mathrm{O}_{2}$ flow are independent. However, with fluorocarbon gases, we expect additional effects, e.g., an increase in the power will increase carbon dissociation, thereby increasing $\mathrm{CO} / \mathrm{CO}_{2}$ production which, in turn, leads to a reduction of oxygen density. An increase of $\mathrm{C}_{4} \mathrm{~F}_{8}$ flow will increase the energy cost per electron-ion pair, thus reducing plasma density.

The proposed model includes the assumption that an ion flux change will generate a change in the same direction for atomic and molecular oxygen densities but will have an opposite effect on the amount of $\mathrm{CO}$. The innovation in this approach is including a setpoint as the part of model, allowing us to modify the requested density, according to the value obtained from the model. In this way we get a value of ion flux or density of oxygen or $\mathrm{CO}$ commensurate with the original set point, with less offset and settling time.

On the following graphs, the controlled outputs of $\mathrm{O}, \mathrm{O}_{2}$, and $\mathrm{CO}$, with and without multivariable control, are presented for different experimental conditions. Figure 6 shows the atomic oxygen measurement with both a low and high contribution of $\mathrm{C}_{4} \mathrm{~F}_{8}$. We request the system to follow the set point from the model (the dotted line) resulting in a measured atomic oxygen signal very close to the real setpoint and with minimal delay. Since the flows of other gases like $\mathrm{C}_{4} \mathrm{~F}_{8}$ or Ar are not included in the closed-loop strategy, there can be some disagreement between the real set point and the measured value, even with the use of a model, when the contributions of $\mathrm{C}_{4} \mathrm{~F}_{8}$ very high, as in the case presented on the right side of Fig. 6. However, even in this case, it is evident that the model gives better results than control without the model, with rms error values of 0.0056 and 0.0066 achieved with the model, compared to 0.0141 and 0.0138 without the model, for low and high concentrations of $\mathrm{C}_{4} \mathrm{~F}_{8}$, respectively. This feature is more evident in the case of controlling $\mathrm{O}_{2}$ or $\mathrm{CO}$ densities.

Figure 7, for example, demonstrates the control of molecular oxygen and ion flux. As can been seen from Fig. 7,

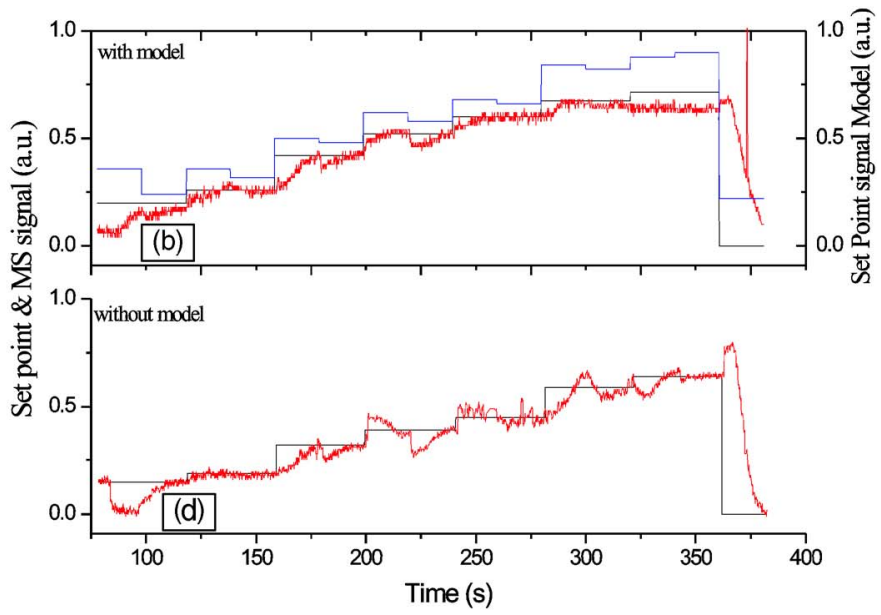

FIG. 6. (Color online) Real-time control of atomic oxygen. The left side of the graph presents measurements at the experimental point No. 2 in Table I. The right side of the graph presents measurements at the experimental point No. 6 in Table I. The solid line shows the real set point, the broken line represents the measured value of $(\mathrm{O})$, and the dotted line is the modified set point derived from the model. 

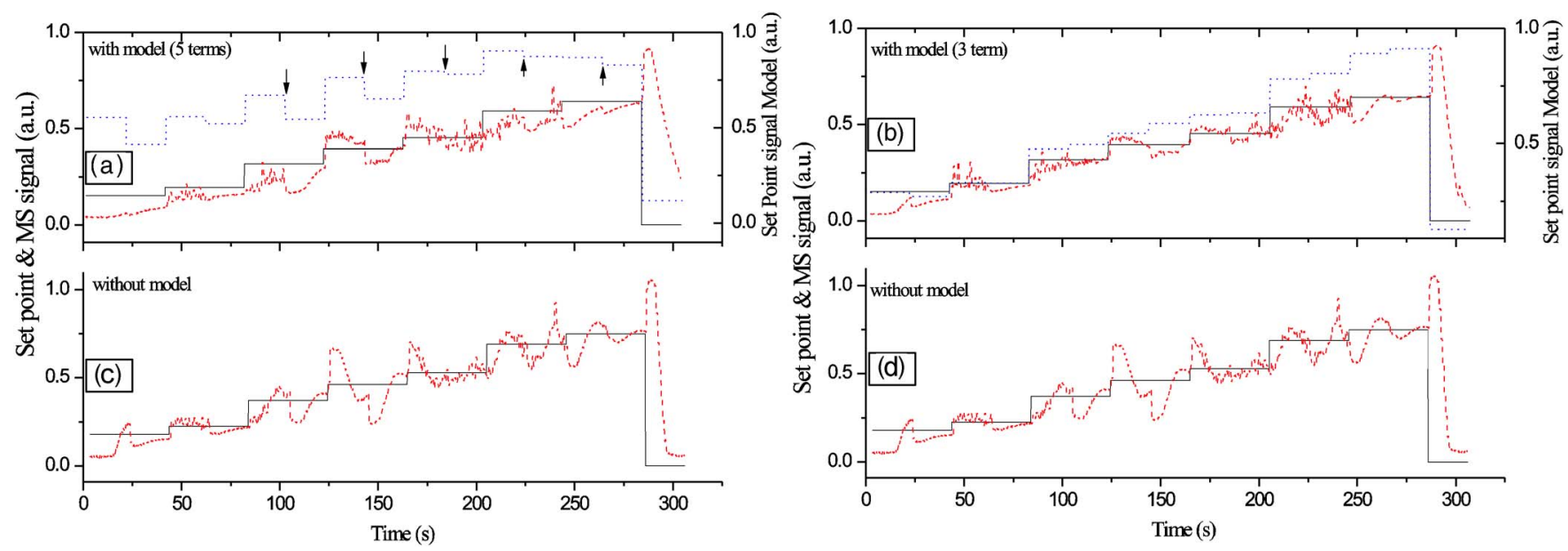

FIG. 7. (Color online) Real-time control of molecular oxygen at the experimental point No. 6 from the Table I. The left side of the graph shows a measurement with a five-term model [see Eq. (1)]. The right side of the graph shows a measurement with a three-term model, i.e., $\hat{y}_{g}=a_{1} r_{g}+a_{2} r_{f}+a_{5} r_{f} r_{g}$. The solid line represents the real set point, the broken line is the measured $\mathrm{O}_{2}$ value, and the dotted line is the modified set point derived from the model.

models improve the agreement between the measured and real set point values in comparison with no model usage (rms error values of 0.0299 compared to 0.0600 for $b$ versus $d$ ). However, the agreement between the measured and real set point values, even with the model, is poor in the case of molecular oxygen (Fig. 7), in comparison with the control of atomic oxygen (Fig. 6). The main reason for that is the existence of oxygen in the solid phase, i.e., the wafer is composed of $\mathrm{SiO}_{2}$ and when the plasma is struck. The chamber contains molecular oxygen, not only from the gas feed (which we have active control over), but also as an etch product of the $\mathrm{SiO}_{2}$ surface. Without the discharge, no signal appears at mass 28 , but as soon as the discharge is struck, we measure a significant signal on mass 28 ( $\mathrm{Si}$ and/or $\mathrm{CO})$. In Fig. 7(a), five arrows are shown, signifying the moment when the modified set point has changed (the dotted line), but the real set point (the solid line) remains the same. The model requests a change in the set point even when the real set point is not changed due to the change in ion flux set point (Fig. 5). This confirms the hypothesis that oxygen in our discharge comes from both the gas feed and the wafer.
Figure 7(b), shows results for a modified model, omitting the $a_{3} r_{g}^{2}$ and $a_{4} r_{f}^{2}$ terms. The modified model reduces the influence of changing the set point of ion flux on the control of molecular oxygen. With this three-term model, shown in Fig. 7(b), we get closer agreement between the measured value and real set-point value, compared to the five-term model [Fig. 7(a)]. Nevertheless, the total control of molecular oxygen requires not only active control over the mass flow controller for oxygen but also "control" of the emission of oxygen from the wafer. The problems with this secondary oxygen emission are more evident in the case of the control of $\mathrm{CO}$ density.

Figure 8 presents the control of carbon monoxide (CO) for two different experimental conditions. Control of $\mathrm{CO}$ density is more difficult than control of molecular oxygen as we do not have active control over any carbon species. The availability of carbon for the formation of $\mathrm{CO}$ is entirely dependent on the plasma. Indirect control of carbon is possible via control of the reactants on the left-hand side of Eq. (2), where P27 roughly maps onto electron density and $\mathrm{O}_{2}$ flow onto $\mathrm{O}_{2}$ density. Carbon exists both in the gas phase in
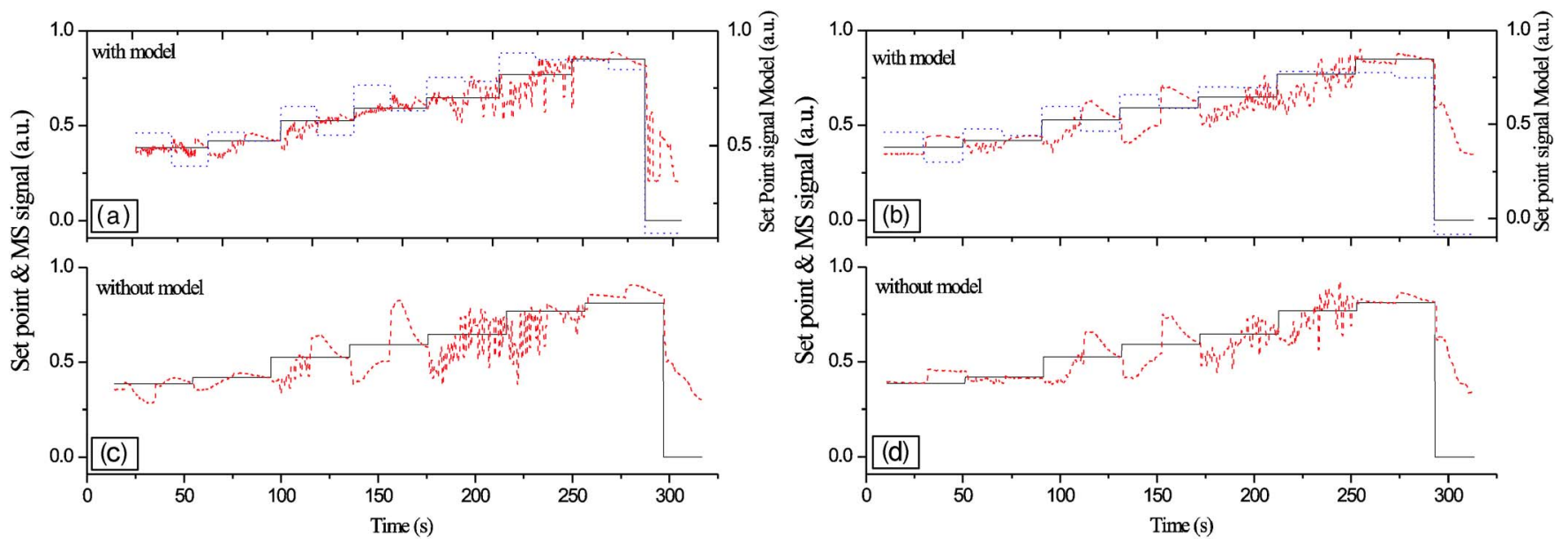

FIG. 8. (Color online) Real-time control of CO density. The left side of the graph presents measurements at experimental point No. 6 in Table I. The right side of the graph shows measurement at experimental point No. 16 in Table I. The solid line is the real set point, the broken line is the measured value, and the dotted line is the modified set point derived from the model. 
the $\mathrm{C}_{4} \mathrm{~F}_{8}$ molecule and also in the solid phase as fluorocarbon residue on the walls of the discharge chamber from previous process steps. Sputter rates are proportional to ion flux, however, variations in the low frequency $(2 \mathrm{MHz}) \mathrm{rf}$ power leads to changes in ion energy on chamber walls, and thus sputtering of any carbon-containing film. Therefore, we can only partially control the emission of carbon atoms from the walls. Nevertheless, we demonstrate reasonably good control of CO density by use of the model, in comparison to without use of the model (left side of the Fig. 8), when the influence of a perturbation such as power of $2 \mathrm{MHz}$ rf generation or flow of $\mathrm{C}_{4} \mathrm{~F}_{8}$ is diminished (rms errors of 0.0554 compares to 0.0428). For the case of a large perturbation in, for example, $2 \mathrm{MHz}$ rf power or flow of $\mathrm{C}_{4} \mathrm{~F}_{8}$, it is very difficult to keep the $\mathrm{CO}$ density around the real set point, even with model usage, resulting in comparable rms errors with and without model usage.

One possible solution for better control of $\mathrm{CO}$ density could be to have active control of the $2 \mathrm{MHz}$ rf generator and the $\mathrm{C}_{4} \mathrm{~F}_{8}$ mass flow controller. It is important to point out that control of carbon, via control of the $\mathrm{C}_{4} \mathrm{~F}_{8}$ mass flow controller, is not straightforward. In any case, extending the active multivariable control will give better control of CO density, but the complexity of the model will increase significantly. Obviously, it is necessary to find a compromise between complexity of the model and the level of control, for different plasma species.

\section{CONCLUSION}

In this paper, we demonstrate real-time independent control of ion flux and plasma chemistry $\left(\mathrm{O}, \mathrm{O}_{2}\right.$, or $\left.\mathrm{CO}\right)$ in a modified Exelan ${ }^{\circledR}$ chamber (Lam Research). The control is reasonably insensitive to external perturbations in the operating point of the chamber. The ion flux is measured via an isolated collection area built into the electrode surface, biased to $-18 \mathrm{~V}$. Density of chemical species is measured using mass spectrometry or OES, via actinometry. The response surface of the sensors in the process space was collected over the process space. The control of two independent variables (ion flux and density of atomic/molecular species) in real time is initially performed using two single-loop PID controllers. By modeling the plasma parameters over a desired operational space, a simple physics-based model of the interaction is generated. We show that, in an $\mathrm{Ar} / \mathrm{O}_{2} / \mathrm{C}_{4} \mathrm{~F}_{8}$ plasma, by employing the physics-based model in a multivariable control scheme, superior control results.

Ion flux and species density signals are not normally orthogonal, and do not (respectively) directly map onto the input control variables of rf power and gas flow. The output signals are compared to external set points for ion flux and chemical density. The functional dependencies of the response surface, in conjunction with simple physical models, are used to deconvolve the sensor signals onto the control parameters.

We have also developed a multiple set of model parameters across the space of process operation, which allows us to schedule the appropriate model parameters in conjunction with recipe changes. However, the model-based control scheme has also shown good robustness to process variations, even when model parameters have been fixed. The model therefore shows good reproducibility and control, and can reliably be used for etch/deposition control.

We have custom designed the optical devices to avoid using an external software driver. With this optical device, we directly measure an analog signal, therefore avoiding conversion from the digital to the analog domain. In addition, the optical filters have better resolution than many commercial spectrometers. The ion-flux probe is specially designed to measure only positive ions and is of a design for robust implementation in manufacturing.

The model-based control gives very good results for the control of atomic oxygen. Control of molecular oxygen is more difficult, possibly due to the additional oxygen in the gas chemistry arising from the etching of the $\mathrm{SiO}_{2}$ wafer. Nevertheless, even in this case, we get reasonably good control of $\mathrm{O}_{2}$ by changing the number of terms in the model. Control of $\mathrm{CO}$ density represents the most significant challenge. The main reason for this is the inability to directly control carbon. We do have indirect control of carbon emission, i.e., we control the ion flux and therefore control of etching or dissociation. The final control topology therefore represents a complexity/performance compromise between number of control inputs and the number of controlled physical parameters.

\section{ACKNOWLEDGMENTS}

This work is a part of Project No. 02/IN.1/I147 funded by Science Foundation Ireland and European Union funding under EURATOM Contract No. FU06-CT-2004-00068 and project "The use of non invasive OES as method for the determination of gas species concentration and the main plasma parameters in industrial plasma processing equipment" funded by Enterprise Ireland. V. Milosavljević is grateful to the Ministry of Science and Environment Protection of the Republic of Serbia for Grant No. OI141031 "Nonlinear dynamical phenomena in photorefractive media, liquid crystals, plasmas, and left-handed materials."

${ }^{1}$ P. C. Boyle, A. R. Elligboe, and M. M. Turner, J. Phys. 37, 697 (2004).

${ }^{2}$ J. Robiche, P. C. Boyle, M. M. Turner, and A. R. Ellingboe, J. Phys. D 36, 1810 (2003).

${ }^{3}$ J. P. Booth, H. Abada, P. Clabert, and D. B. Graves, Plasma Sources Sci. Technol. 14, 273 (2005).

${ }^{4}$ J. P. Booth, O. Joubert, J. Pelletier, and N. Sadeghi, J. Appl. Phys. 69, 618 (1991).

${ }^{5}$ J. P. Booth and N. Sadeghi, J. Appl. Phys. 70, 611 (1991).

${ }^{6}$ G. S. Oehrlein, P. J. Matsuo, M. F. Doemling, N. R. Rueger, B. E. E. Kastenmeier, M. Schaepkens, Th. Standaert, and J. J. Beulens, Plasma Sources Sci. Technol. 5, 193 (1996).

${ }^{7}$ Lam Research, Applied Materials and Hitachi, private communication (2007).

${ }^{8}$ T. F. Edgar, S. W. Butler, W. J. Campbell, C. Pfeiffer, C. Bode, S. B. Hwang, K. S. Balakrishnan, and J. Hahn, Automatica 36, 1567 (2000).

${ }^{9}$ S. W. Butler, K. J. McLaughlin, T. F. Edgar, and I. J. Trachtenberg, J. Electrochem. Soc. 138, 2727 (1991).

${ }^{10}$ B. A. Rashap, M. E. Elta, H. Etemad, J. P. Fournier, J. S. Freudenberg, M. D. Giles, J. W. Grizzle, P. T. Kabamba, P. P. Khargonekar, S. Lafortune, J. R. Moyne, D. Teneketzis, and F. L. Terry, Jr., IEEE Trans. Semicond. Manuf. 8, 286 (1995).

${ }^{11}$ J. Waddington and F. Fallside, Int. J. Control 3, 1 (1966).

${ }^{12}$ O. D. Nwokah, R. E. Nordgren, and G. S. Grewal, IEE Proc.: Control 
Theory Appl. 142, 23 (1995).

${ }^{13}$ S. K. Karkari and A. R. Ellingboe, Appl. Phys. Lett. 88, 101501 (2006).

${ }^{14}$ V. Milosavljević and A. R. Ellingboe, PRL internal report, Dublin City University, Dublin, 2004).

${ }^{15}$ V. Milosavljević, R. Faulkner and M. B. Hopkins, Opt. Express 15, 13913
(2007).

${ }^{16}$ F. W. Breitbarth, E. Ducke, and H. J. Tiller, Plasma Chem. Plasma Process. 10, 377 (1990)

${ }^{17}$ A. Granier, D. Chereau, K. Henda, R. Safari, and P. Leprince, J. Appl. Phys. 75, 104 (1994). 